\title{
CONSUMO DE POTENCIA EN UNIDADES DE FLUIDIZACION DE ASERRIN
}

\author{
Rogelio Moreno M. $\left(^{*}\right)$ \\ Rolando Ríos R. (*) \\ Heriberto Soto C. (**) \\ Floriano Núñez P. (***)
}

\section{RESUMEN}

El objetivo de este trabajo consiste en evaluar la potencia eléctrica consumida por el sistema de impulsión de aire en unidades de fluidización de aserrín.

Los resultados han sido obtenidos a través de experiencias de laboratorio relacionadas con la suspensión de partículas de aserrín, diseño de distribuidores de aire y diseño y construcción de un soplador centrífugo de aire con velocidad variable.

Los resultados permiten además, determinar las características de diseño del sistema de impulsión de aire y los costos operacionales por consumo de potencia eléctrica de una unidad de fluidización de aserrín, ya sea para la combustión, el secado o la gasificación de este desecho forestal.

Palabras claves: Aserrin, Consumo de potencia, Energia, Fluidización.

(*) M. Sc., Ingeniero Civil Mecánico

(*) M. Sc., Ingeniero de Ejecución Mecánico

(**) M. Sc., Ingeniero de Ejecución Electrónico, Instituto de Electricidad y Electrónica Facultad de Ciencias de la Ingenierla, Universidad Austral de Chile, Casilla 567, Valdivia.

(*..*) Alumno memorista Instituto de Materiales y Procesos Termomecánicos 


\section{ABSTRACT}

The aim of this work is to evaluate the electrical power consumed by air drive systems in sawdust fluidization units.

The results have been obtained through laboratory work concerning the suspension of sawdust particles, design of air distributors, as well as design and construction of a centrifugal air blower of variable speed.

Furthermore, the results allow to determine not only the design characteristics of the air drive system, but also the operational costs coming from the consumption of electrical power in a sawdust fluidization unit, whether it is for combustion, drying or gasification of this forest waste.

Keywords: Sawdust, Power consumption, Energy, Fluidization. 


\section{INTRODUCCION}

La creciente actividad del sector forestal en la zona sur de Chile ha generado una gran acumulación de desechos forestales derivados de la explotación y elaboración de la madera. A mediados de la década anterior, la generación anual de estos residuos industriales era de 3 millones de toneladas (Wagemann, 1984). Según cifras más recientes (Méndez et al., 1994) y basadas en informes técnicos del Instituto Forestal, el $83 \%$ de los 7 millones de toneladas de madera explotadas, corresponde a pino insigne. Uno de los desechos más visibles es el aserrín, el cual despierta preocupación debido a los altos volúmenes acumulados y a las superficies de suelos que se han ido cubriendo y contaminando a velocidades cada vez mayores.

A pesar de la gran cantidad de calderas que hay actualmente en el pais para el quemado de desechos forestales, el volumen acumulado de ellos sigue creciendo. Es importante, por tanto, seguir estudiando altemativas atractivas para darles un uso racional. El empleo de métodos de combustión en parrilla junto a la gran humedad del combustible, ha conducido, tradicionalmente, al diseño de calderas de gran tamaño.

En la actualidad, el empleo de la fluidización, como tecnologia apta para el quemado de sólidos en suspensión, es una realidad a nivel mundial, debido a sus ventajas técnicas, relacionadas con las altas tasas de transferencia de calor y de combustión y a la posibilidad de controlar las emisiones de particulas contaminantes al ambiente en el mismo reactor. Es notable el desarrollo científico y tecnológico que dicha tecnología ha tenido a partir de los años 70 (Fennelly, 1984). Particularmente en los Estados Unidos, este desarrollo ha sido posible gracias a fuertes apoyos financieros gubernamentales para la realización de investigaciones y estudios en equipos de laboratorio, plantas piloto y plantas industriales.

Hoy en dia, las capacidades de las calderas de carbón en lecho fluidizado superan los $110 \mathrm{~kg} / \mathrm{s}$ de producción de vapor, con alta captación de $\mathrm{SO}_{2}$ (sobre el $90 \%$ ) y baja formación de $\mathrm{NO}_{x}$ (inferior a $300 \mathrm{Vppm}$ ); las eficiencias térmicas, por otro lado, son del orden de $90 \%$. En los países nórdicos, también se ha conseguido desarrollar calderas de lecho fluidizado para la combustión de 
desechos forestales con humedades de $65 \%$ y capacidades de hasta $72 \mathrm{~kg} / \mathrm{s}$. En Brasil y Cuba la tecnologia es empleada para quemar bagazo de caña.

La experiencia en el país es reciente; sin embargo, a la fecha existe una planta piloto, diseñada y construida para el quemado de carbones nacionales de alto contenido de cenizas (Navarrete et al., 1992). Actualmente, el estudio se desarrolla para el quemado de desechos forestales. Resultados recientes sobre tasas de transferencia de calor (Navarrete et al., 1993; Navarrete et al., 1994, Moreno et al., 1994), revelan que efectivamente con carbones nacionales y con desechos forestales, es posible llegar a un diseño de calderas más compactas y económicamente más atractivo. También existen en el país resultados preliminares sobre gasificación de aserrín en lecho fluidizado (Méndez et al., 1994), los cuales han conducido a un poder calorífico del gas de $2,77 \mathrm{MJ} / \mathrm{Nm}^{3}$.

Una de las desventajas que normalmente aparecen citadas en la literatura para las unidades de lecho fluidizado, es el consumo de energía del sistema impulsor de aire al reactor (Kunii y Levenspiel, 1969). Por tal motivo, previo a tomar una decisión sobre el diseño de una unidad de este tipo, se debe efectuar un estudio sobre el consumo de potencia eléctrica del equipo y los costos operacionales que se derivan de él.

\section{OBJETIVO}

Este trabajo tiene por objetivo dar a conocer los parámetros que intervienen en el cálculo de la potencia consumida por el sistema de impulsión de aire de una unidad de fluidización, aplicado al caso particular del aserrín. El trabajo se basa en resultados experimentales sobre velocidades de operación y pérdidas de presión en un lecho fluidizado de aserrín, experiencias sobre distribuidores de aire tipo multiorificios y resultados obtenidos en el diseño y construcción de un sistema de impulsión de aire para una columna de fluidización. El sistema de impulsión se compone de un soplador centrifugo y un motor dotado de un regulador de velocidad. Esto último, también ha permitido efectuar estudios de ahorro de energía. 


\section{MATERIAL Y METODO}

\section{Equipo y Material}

Se diseña y construye una unidad de fluidización de aserrín de geometria rectangular, de $0,5 \times 0,1 \mathrm{~m}$, según se muestra en la Figura $\mathrm{N}^{\circ} 1$. El distribuidor consiste de un plato multiorificios. El sistema de impulsión de aire consta de un soplador centrifugo, con un motor de arrastre de 5,59 kW, de velocidad variable. Las velocidades del aire se determinaron a partir de datos proporcionados por un medidor de flujo, ubicado en la tuberia de alimentación de aire al reactor. Las pérdidas de carga se midieron con manómetros diferenciales de agua.

Los sólidos ensayados son partículas de aserrín de pino insigne, cuyo tamaño medio oscila entre 1,59 y 3,56 × $10^{-3} \mathrm{~m}$, clasificados granulométricamente por medio de tamizado ASTM. La operación de un combustor 0 un gasificador fluidizado requiere de la introducción de sólidos inertes, tales como arena o ceniza para la mantención y estabilización de las temperaturas de operación. Por tal motivo, en dichas operaciones, los parámetros de diseño de estas unidades deben considerar que en el interior del reactor habrá una mezcla de sólidos combustibles e inertes. En esta investigación se trabajó con arena como sólido inerte, con un tamaño de particula de 0,3 a $1,18 \times 10^{-3} \mathrm{~m}$. 


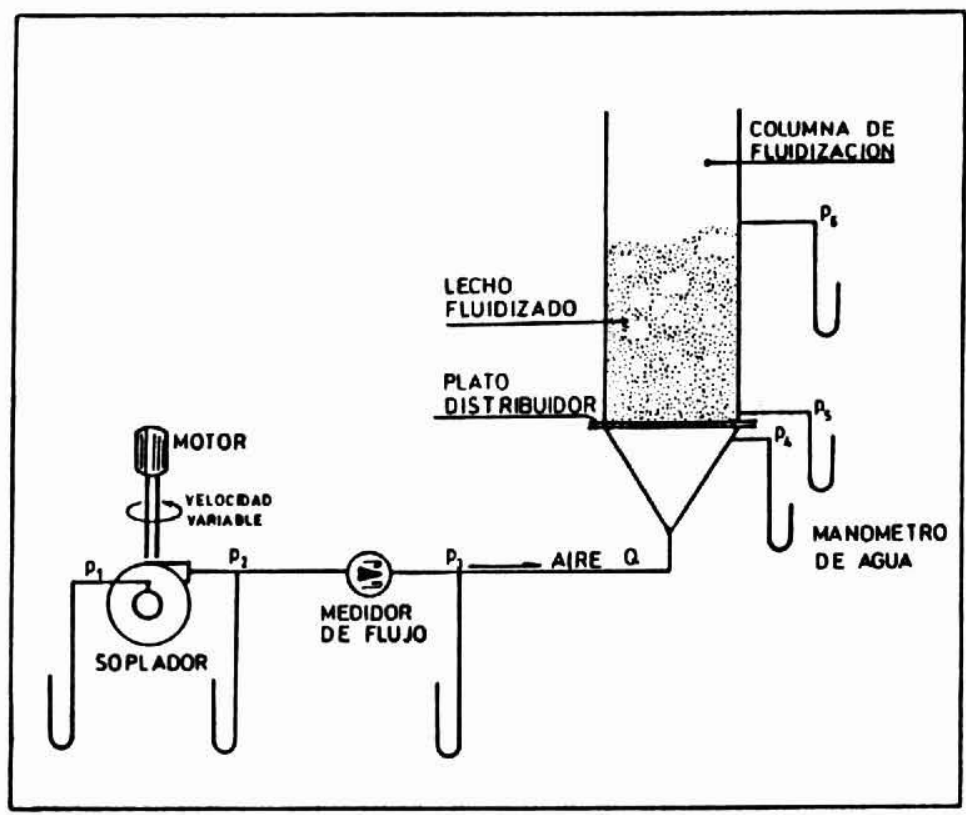

Figura No 1. DIAGRAMA ESQUEMATICO DEL EQUIPO EXPERIMENTAL

\section{Metodologia}

La potencia absorbida por el equipo impulsor depende de la presión estática y del caudal requerido para la operación de la unidad. La presión estática, a su vez, depende de las pérdidas de carga que se producen en el lecho, en el distribuidor de aire y en las tuberias de alimentación de aire. Por otro lado, el caudal de impulsión depende de la velocidad de operación de la unidad de fluidización; ésta queda determinada entre la velocidad minima de fluidización y la velocidad de arrastre de los sólidos. En la Figura № 1, además de los componentes del equipo, se muestran los parámetros involucrados en el cálculo de la potencia hidráulica del equipo impulsor, a través de la ecuación (1), la cual corresponde a la potencia teórica para una operación adiabática reversible y considerando al aire como un gas perfecto (Kunii y Levenspiel, 1969) 


$$
N_{h}=\frac{\gamma}{\gamma-1} \frac{p_{2} Q_{2}}{3600}\left[1-\left(\frac{p_{1}}{p_{2}}\right)^{\frac{\gamma-1}{\gamma}}\right]
$$

donde $\gamma=C_{p} / C_{v}$.

La potencia al eje, absorbida por el equipo impulsor, será la potencia teórica afectada por el rendimiento mecánico de la máquina, el cual, según Kunii y Levenspiel, debería oscilar entre 55 y $75 \%$, para turbosopladores. Finalmente, la potencia consumida por el motor eléctrico será la potencia absorbida por el impulsor dividida por el rendimiento eléctrico del motor.

$$
\begin{aligned}
& N_{a}=\frac{N_{h}}{\eta_{m}} \times 100 \\
& N_{e}=\frac{N_{a}}{\eta_{e}} \times 100
\end{aligned}
$$

Para el cálculo de la potencia hidráulica, se deberá determinar previamente el valor de $p_{2}$, según:

$$
p_{2}=p_{6}+\Delta p_{\text {lecho }}+\Delta p_{\text {dist }}+\Delta p_{\text {tub }}
$$




\section{RESULTADOS Y DISCUSION}

En el Cuadro $N^{\circ} 1$ se muestra el valor de la pérdida de carga unitaria del lecho de partículas de aserrín, para tres tamaños de partícula.

\section{Cuadro $\mathrm{N}^{\circ} 1$}

PERDIDA DE CARGA UNITARIA DEL LECHO DE PARTICULAS DE ASERRIN

\begin{tabular}{|c|c|}
\hline$d_{p}(\mathrm{~m})$ & $\Delta \mathrm{P}_{\text {hecho }} / \mathrm{H}(\mathrm{Pa} / \mathrm{m})$ \\
\hline 0,00159 & 1369 \\
\hline 0,02180 & 1203 \\
\hline 0,03560 & 1094 \\
\hline
\end{tabular}

Los valores del Cuadro $\mathrm{N}^{\circ} 1$ fueron medidos directamente en el lecho de partículas de aserrín, cuyo contenido de humedad era de $23,5 \%$ base seca. La determinación de la pérdida de carga del lecho se ha efectuado con una velocidad superficial del aire igual o superior a la velocidad mínima de fluidización, condición en la cual la mayor parte de los sólidos se encuentra en suspensión (Moreno et al., 1994). Debido a que la humedad de los sólidos influye directamente en el peso de ellos y en base a experiencias realizadas con otro tipo de desechos forestales (Moreno et al., 1993), que revelan que el aumento de la pérdida de carga del lecho con la humedad es de tipo lineal, los valores del Cuadro $\mathrm{N}^{\circ} 1$ se pueden corregir empleando la ecuación (5) (Davidson y Harrison, 1963). La densidad de los sólidos, en el caso particular del aserrín, se calcula en función de la gravedad específica de la especie maderera de la cual proviene y su humedad base seca, con la ecuación (6).

$$
\begin{aligned}
\Delta p_{\text {lecho }} & =H(1-\varepsilon)\left(p_{s}-p_{a}\right) g \\
p s & =\frac{G S(100+H M)}{100}
\end{aligned}
$$


De esta manera, se puede determinar el parámetro $\Delta p_{\text {lecho, }}$ para cualquier altura de lecho y humedad de aserrín, con la ayuda de la Figura $\mathbf{N}^{\circ} \mathbf{2}$.

La introducción de sólidos inertes en el lecho es una práctica necesaria y habitual en operaciones tales como la combustión y gasificación en lecho fluidizado. Por tal motivo, el consumo de potencia por concepto de fluidización de partículas se ve afectado en función de las variaciones de pérdida de carga que se experimenten con sólidos de diferente densidad o peso. Dado que uno de los sólidos inertes más empleados en los procesos mencionados es la arena, se efectuaron ensayos de fluidización con este material y los resultados obtenidos se muestran en el Cuadro $\mathrm{N}^{\circ} 2$.

\section{Cuadro $\mathrm{N}^{\circ} 2$}

PARAMETROS PARA LA FLUIDIZACION DE ARENA

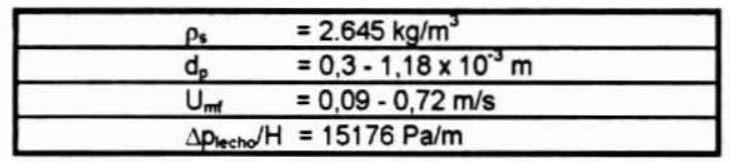

En relación al diseño de distribuidores de aire tipo multiorificios, éste se ha efectuado atendiendo el método propuesto por Richardson (1961); se han construido distribuidores con diferentes características, llegándose a la conclusión que aquél que presenta una fracción de área libre de $5 \%$, proporciona las mejores condiciones para la fluidización (Moreno et al., 1994). La Figura $N^{\circ} 3$ muestra la variación de la pérdida de carga del distribuidor, en función de la velocidad del aire y para diferentes porcentajes de área libre. 


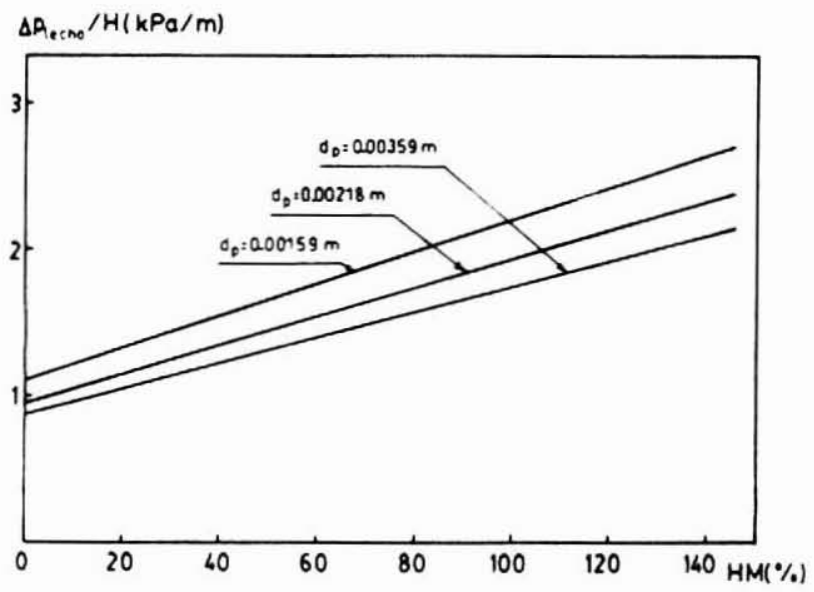

Figura $N^{\circ}$ 2. VARIACION DE LA PERDIDA DE CARGA DEL LECHO DE ASERRIN EN FUNCION DE LA HUMEDAD DE LOS SOLIDOS.

Respecto de las pérdidas de carga en las cañerias de impulsión de aire, éstas se pueden determinar a través de métodos clásicos encontrados en textos de mecánica de fluidos. Sin embargo, la colocación de algún elemento especial en las cañerías, puede dar origen a pérdidas adicionales. Asi por ejemplo, el instrumento de medición de flujo de aire empleado en esta investigación, posee pérdidas como las señaladas en la Figura $\mathrm{N}^{\circ} 4$, para diferentes diámetros.

De este modo, con los resultados anteriores, es posible evaluar con muy buena aproximación el consumo de potencia del impulsor de aire. Para tales efectos, se requiere fijar el caudal de aire, el cual dependerá de la velocidad de operación de la unidad de fluidización. Esta velocidad debe estar entre la velocidad mínima de fluidización y la de arrastre de los sólidos. De acuerdo a resultados previos, con $2,0 \mathrm{~m} / \mathrm{s}$ se pueden fluidizar particulas secas de hasta 4 $\times 10^{-3} \mathrm{~m}$. Sin embargo, en algunas operaciones se trabaja con particulas húmedas y de tamaño variable. Por tal motivo, el consumo de energia será variable según la condición de operación. 


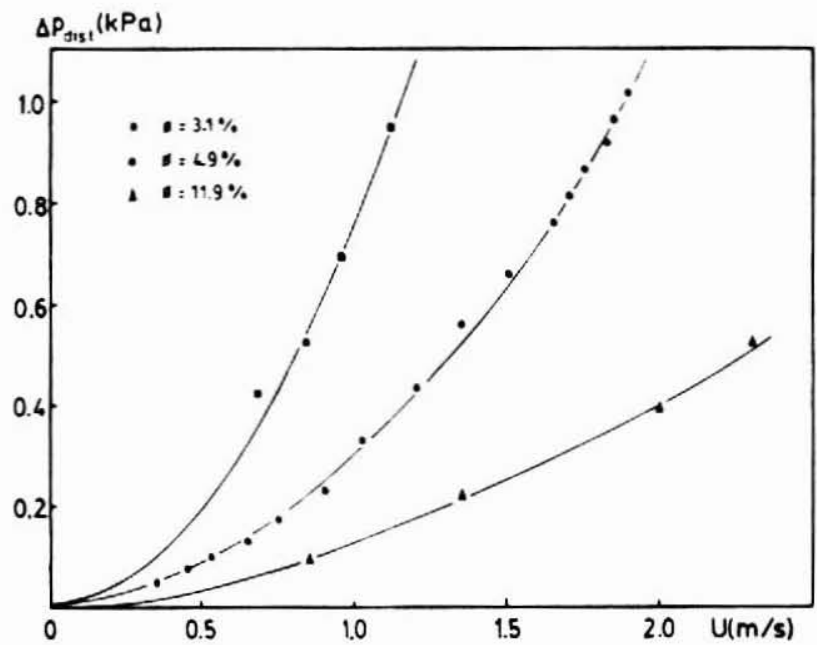

Figura $N^{\circ}$ 3. PERDIDA DE CARGA DEL DISTRIBUIDOR EN FUNCION DE LA VELOCIDAD SUPERFICIAL DEL AIRE.

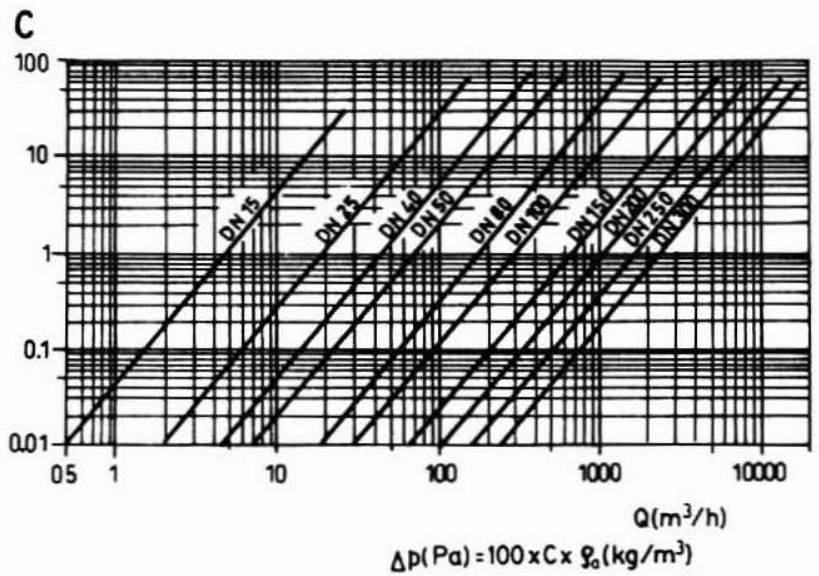

Figura $N^{\circ}$ 4. PERDIDA DE CARGA EN MEDIDOR DE FLUJO EN FUNCION DEL CAUDAL DE AIRE. 
En las Figuras $N^{\circ} 5$ y 6 se muestran los consumos de potencia correspondientes a la suspensión de particulas de aserrín, con una humedad $23,5 \%, y$ al distribuidor, respectivamente, en función de la velocidad de operación.

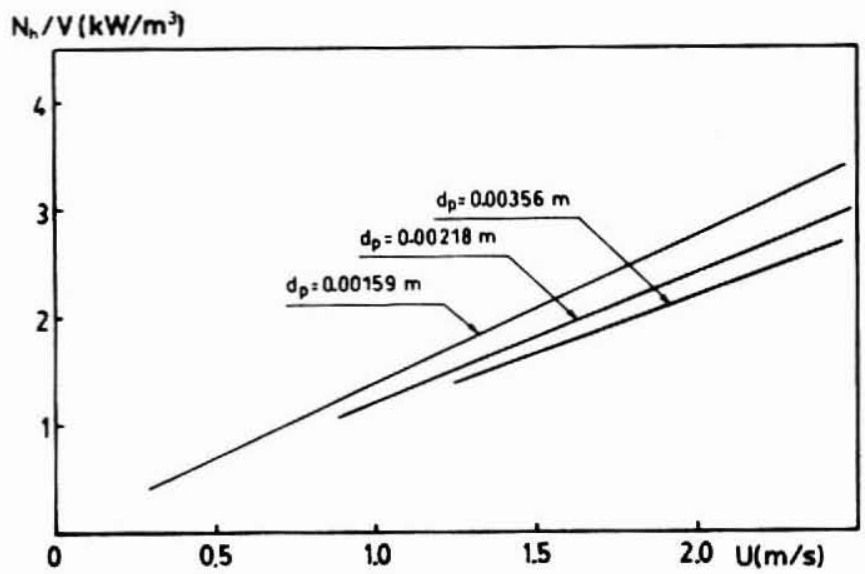

Figura $N^{\circ} 5$. POTENCIA HIDRAULICA PARA LA FLUIDIZACION DE ASERRIN SECO POR UNIDAD DE VOLUMEN DE LECHO EN FUNCION DE LA VELOCIDAD SUPERFICIAL DEL AIRE.

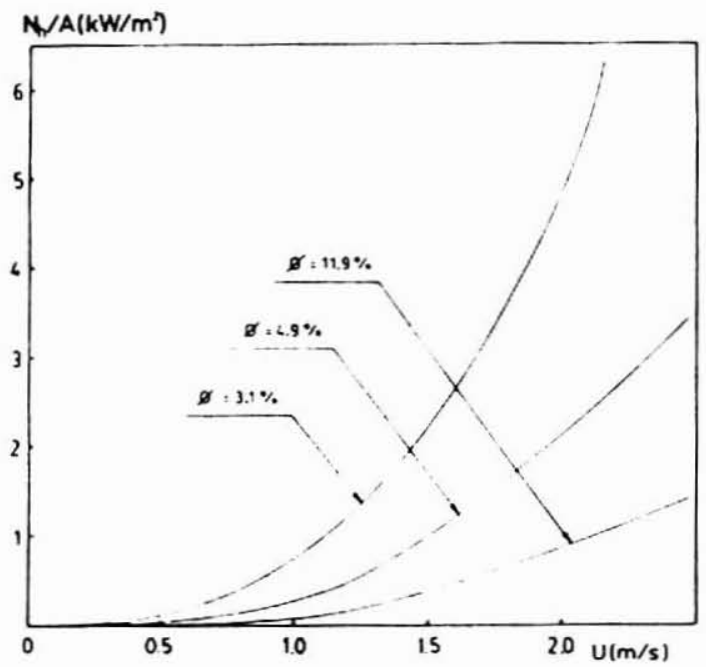

Figura Nº. POTENCIA HIDRAULICA CONSUMIDA EN EL DISTRIBUIDOR EN FUNCION DE LA VELOCIDAD SUPERFICIAL DEL AIRE. 
En base a las velocidades de operación y pérdidas de carga del lecho de partículas de arena, mostradas en el Cuadro $\mathrm{N}^{\circ} 2$, el consumo de potencia correspondiente a la suspensión de ellas, se obtiene empleando las ecuaciones (1) y (4), al igual que en el caso del aserrín.

Finalmente, además de los consumos de potencia, los resultados obtenidos, particularmente los relativos a velocidades y pérdidas de presión, permiten determinar el tipo de máquina de impulsión a emplear. De acuerdo a la relación caudal-presión obtenida en este estudio, la cual se verá reflejada más adelante en la Figura $\mathrm{N}^{\circ} 7$, el equipo más apto es un soplador centrífugo. A este respecto, la investigación contempló el diseño y construcción de un soplador de aire con un motor dotado de un regulador de velocidad.

El ensayo del equipo dio como resultado un rendimiento de $50 \%$, para el conjunto motor-soplador, por lo que el rendimiento mecánico del soplador es cercano al $60 \%$, para un caudul de operación de $1630 \mathrm{~m}^{3} / \mathrm{h}$ y una presión estática de $4570 \mathrm{~Pa}$. En las Figuras $\mathrm{N}^{\circ} 7$ y 8 , se muestran las curvas de presión estática del soplador y de consumo de potencia eléctrica del motor, respectivamente, en función del caudal de aire. Los ensayos se efectuaron para una velocidad nominal de $3000 \mathrm{rpm}$ y a velocidad variable. En este último caso, se empleó un variador de velocidad que actúa sobre el motor a través de la frecuencia de la energía eléctrica que lo alimenta. La relación obtenida, entre velocidad de giro versus frecuencia, es del tipo lineal, con una velocidad máxima de $3000 \mathrm{rpm}$ para una frecuencia de 50.

De estos resultados, se ha podido concluir lo importante que resulta operar el sistema impulsor con un regulador de velocidad, cuando los requerimientos de caudal son inferiores a los de diseño. Los ahorros de energía son significativos frente a la alternativa de emplear un amortiguador de caudal en la descarga del soplador y el sistema motor-soplador trabajando a velocidad nominal, tal como lo muestra la Figura $\mathrm{N}^{\circ} 8$. 


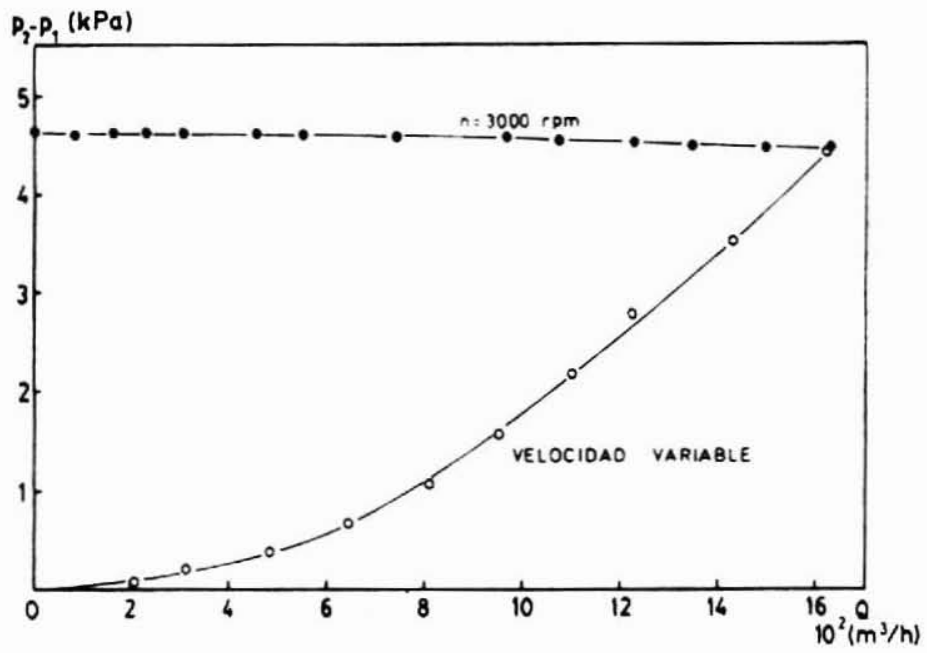

Figura $N^{\circ}$ 7. PRESION ESTATICA DEL SOPLADOR EN FUNCION DEL CAUDAL DE AIRE.

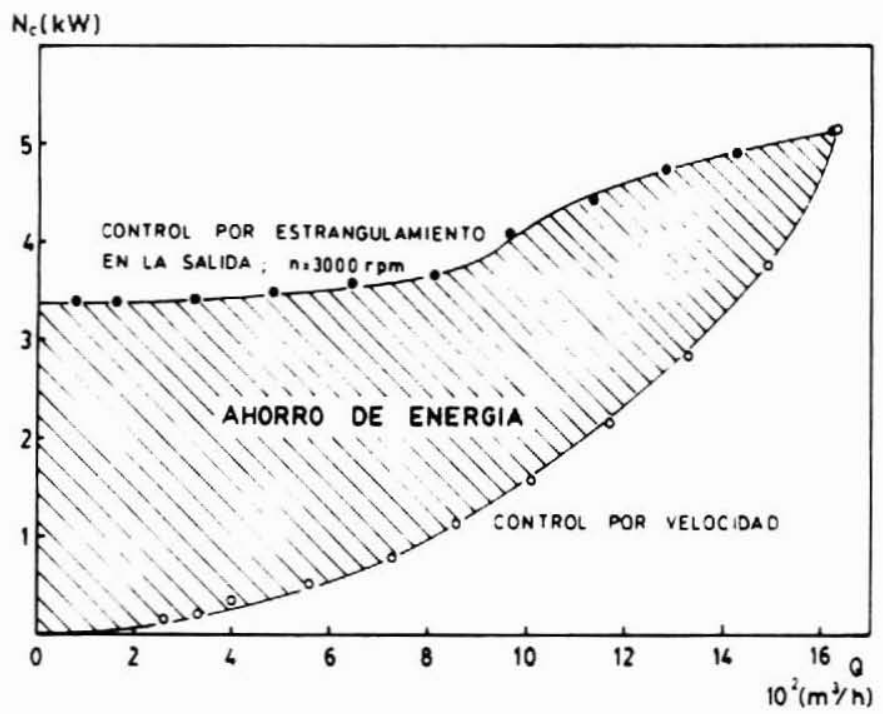

Figura $N^{\circ}$ 8. CONSUMO DE POTENCIA ELECTRICA DEL MOTOR EN FUNCION DEL CAUDAL DE AIRE. 


\section{CONCLUSIONES}

En base a experiencias de laboratorio, se proporcionan los parámetros que permiten determinar las caracteristicas del sistema de impulsión de aire para una unidad de fluidización de aserrín.

De acuerdo a los valores obtenidos para la presión estática versus caudal de aire, se concluye que el tipo de máquina a emplear debe ser un soplador centrífugo. Estos resultados han sido obtenidos empleando distribuidores de aire tipo multiorificios.

El estudio ha permitido proporcionar conclusiones respecto de los consumos de potencia eléctrica del sistema. Los costos operacionales involucrados en la operación del proceso, se obtienen multiplicando el valor de los kW-h consumidos en un periodo determinado por el costo unitario de la energía eléctrica.

También, se ha podido concluir que es altamente recomendable dotar al motor del soplador de un regulador de velocidad, con el fin de ahorrar energia en caso de utilizar el sistema de impulsión a carga parcial.

\section{RECONOCIMIENTO}

Este trabajo es parte del proyecto FONDECYT 1930357. Los autores agradecen a CONICYT el apoyo financiero otorgado.

\section{REFERENCIAS}

Davidson, J. F. y D. Harrison., 1963. Fluidised Particles, Cambridge University Press, London, 13.

Fennelly, P. F., 1984. Fluidized Bed Combustion, American Scientist, 72: 254-261. 
Kunii, D. y O. Levenspiel., 1969. Fluidization Engineering, John Wiley \& Sons, Inc., New York, 100-103.

Méndez, C., X. García y A. Gordon., 1994. Gasificación de Aserrín en Lecho Fluidizado. Estudio Preliminar, Anales Encuentro Latinoamericano de Ingeniería Química, Antofagasta, 238-243.

Moreno, R., M. Paredes, D. Baeza y A. Améstica., 1993. Comportamiento Hidrodinámico de la Fluidización de Desechos Forestales, Ciencia e investigación Forestal, 7(1): 23-36.

Moreno, R., R. Ríos, H. Soto y M. Paredes., 1994. Relación Mecanistica entre Hidrodinámica y Transferencia de Calor en un Lecho Fluidizado de Aserrín, Información Tecnológica, sometido.

Navarrete, P., U. Zúñiga, F. Valenzuela, L. Faúndez, A. Martinich, A. Jalaf y G. Lira., 1992. Prototipo Modificado de Laboratorio para la Combustión en Lecho Fluidizado, Anales V Congreso Nacional de Ingeniería Mecánica, Punta Arenas, 2:501-505.

Navarrete, P., A. Köhler, L. Faúndez y U. Zúñiga., 1993. Determinación Experimental de Coeficientes de Transferencia de Calor a Tubos Inmersos en Lecho Fluidizado con Combustión de Carbón. Anales XI Jornadas de Transferencia de Calor y Materia, Temuco, 15-20.

Navarrete, P., M. Díaz, U. Zúñiga y L. Faúndez., 1994. Determinación Empírica del Coeficiente Global de Transferencia de Calor para Aire en Tubos con Promotores de Turbulencia en Combustión Fluidizada de Desechos Forestales, Anales XII Jornadas de Transferencia de Calor y Materia, Santiago, 125-131.

Richardson, D. R., 1961. How to Design Fluid-Flow Distributors, Chemical Engineering, 68: 83-86.

Wagemann, G., 1984. Problemas en la Utilización de Desechos de Madera en Chile, Anales Segunda Semana de la Energia, Temuco, 1: 59-69. 


\section{NOMENCLATURA}

A Sección transversal de la columna de fluidización $\left(\mathrm{m}^{2}\right)$

C Constante adimensional

$\mathrm{C}_{\mathrm{p}} \quad$ Calor especifico del aire a presión constante $(\mathrm{J} / \mathrm{kg} \mathrm{K})$

C $\quad$ Calor específico del aire a volumen constante (J/kg K)

$d_{p} \quad$ Tamaño medio de partículas entre dos tamices (m)

GS Gravedad especifica de la especie de los sólidos $\left(\mathrm{kg} / \mathrm{m}^{3}\right)$

g

Aceleración de gravedad $\left(\mathrm{m} / \mathrm{s}^{2}\right)$

H Altura del lecho de particulas sólidas (m)

HM Humedad de los sólidos base seca (\%)

$\mathrm{N}_{\mathrm{a}} \quad$ Potencia absorbida por el soplador (W)

$\mathrm{N}_{\mathrm{e}} \quad$ Potencia eléctrica consumida por el motor (W)

$\mathrm{N}_{\mathrm{h}} \quad$ Potencia hidráulica o teórica (W)

n Velocidad de giro del soplador (rpm)

p Presión de aire $(\mathrm{Pa})$

Q Caudal de aire $\left(\mathrm{m}^{3} / \mathrm{h}\right)$

U Velocidad superficial del aire $(\mathrm{m} / \mathrm{s})$

$U_{m f} \quad$ Velocidad mínima de fluidización $(\mathrm{m} / \mathrm{s})$

V Volumen del lecho de partículas sólidas $\left(\mathrm{m}^{3}\right)$

g Exponente politrópico para un proceso isoentrópico

Dp Caida de presión (Pa) 
e Porosidad del lecho $\left(\mathrm{m}^{3}\right.$ vacio $/ \mathrm{m}^{3}$ lecho)

$\mathrm{h}_{\mathrm{m}} \quad$ Rendimiento mecánico del soplador (\%)

$\mathrm{h}_{\mathrm{e}} \quad$ Rendimiento eléctrico del motor (\%)

$r_{a} \quad$ Densidad del aire $\left(\mathrm{kg} / \mathrm{m}^{3}\right)$

rs Densidad de los sólidos $\left(\mathrm{kg} / \mathrm{m}^{3}\right)$

f Fracción de área libre del distribuidor (\%) 